ISSN 1991-8631

Original Paper

http://indexmedicus.afro.who.int

\title{
Etude de la qualité bactériologique des aliments vendus sur le campus de l'Université d'Abomey Calavi au Bénin
}

\author{
T.A. AHOYO ${ }^{1 *}$, H. AHISSOU ${ }^{2}$, F. KOUNON ${ }^{4}$, T. AMINOU ${ }^{3}$ et K. DRAMANE ${ }^{4}$ \\ ${ }^{I}$ Laboratoire de Biologie Humaine, EPAC / Université d'Abomey-Calavi, BP 2009 Cotonou, Bénin. \\ ${ }^{2}$ Laboratoire de Biochimie, Faculté des Sciences et Techniques, Université d'Abomey-Calavi, 01 BP 526 \\ Cotonou, Bénin. \\ ${ }^{3}$ Laboratoire d'Expertise et de Recherche en Chimie de l'Eau et de l'Environnement (LERCEE), 01 BP 526 \\ Cotonou, Bénin. \\ ${ }^{4}$ Laboratoire de Pharmacodynamie (FAST), 01 BP 526 Cotonou, Bénin. \\ *Auteur correspondant, E-mail: taahoyo@yahoo.fr; BP 2009 Cotonou, Bénin; Tel: (229) 95719015; 90100122
}

\section{RESUME}

Selon l'Organisation Mondiale de la Santé, deux millions de personnes dans les pays en développement décèdent chaque année, à cause des intoxications alimentaires contractées en partie dans la restauration de masse. Le double objectif de cette étude était d'analyser la qualité bactériologique des aliments vendus en restauration rapide sur le campus universitaire et de déterminer la fréquence des bactéries multi résistantes (BMR) isolées. En six mois; 390 échantillons d'aliments ont été collectés et analysés. Escherichia coli, Salmonella, Staphylococcus aureus et Enterococcus ont été recherchées. Les bactéries ont été dénombrées puis identifiées suivant les méthodes biochimiques classiques. Un antibiogramme a été réalisé sur les souches isolées. Le sérotype Escherichia coli $\mathrm{O} 157$ et les souches d'Escherichia coli productrices de bêta lactamase à spectre élargi $(E C B L S E)$ ont été recherchés. $74 \%$ des prélèvements sont contaminés; 325 souches des bactéries recherchées sont isolées, dont 116 (36\%) constituent des BMR parmi lesquelles 44\% de ECBLSE, 32\% de Staphylococcus aureus résistante à la méticilline et $24 \%$ d'Entérocoque résistant à la vancomycine. Deux souches de Salmonella enteritidis et 21 souches d'Escherichia coli $\mathrm{O} 157$ ont été détectées. Il ressort de ces résultats que la qualité bactériologique des aliments analysés n'est pas satisfaisante; la proportion de BMR est élevée, et la transmission aux consommateurs est possible.

(C) 2010 International Formulae Group. All rights reserved.

Mots clés: Toxi-infection alimentaire; BMR, campus universitaire, Bénin

\section{INTRODUCTION}

La restauration collective est devenue un phénomène des sociétés modernes par son importance nutritionnelle et socioéconomique. Mais elle comporte aussi des inconvénients d'ordre sanitaire, parmi lesquels figurent les toxi-infections alimentaires collectives (TIAC) selon Drabo et al. (2009). Ces infections touchent environ $30 \%$ des individus chaque année de part le monde. Près de $80 \%$ des épidémies déclarées proviendraient des restaurants à service rapide «fast-food» selon Chapman et al. (2010). La restauration rapide est florissante en milieu estudiantin dans les universités africaines, d'après le constat de Chauliac et al. (1998). Ceci participe, sans 
doute, au bien-être et au confort des étudiants qui passent toute la journée sur les différents campus universitaires. $\mathrm{Si}$ ce secteur non seulement se maintient, mais continue à se développer dans les villes, c'est qu'il répond à une forte demande des populations urbaines. Il propose à la fois des aliments traditionnels à base de produits locaux, et des plats nouveaux adaptés aux faibles revenus de nombreux résidents urbains (Drabo et al., 2009). Cependant, des flambées de graves maladies infectieuses d'origine alimentaire se sont produites ces dernières années dans plusieurs pays africains, selon un rapport de l'OMS en 2009 et ce, en rapport avec la floraison des restaurants à service rapide. Les TIAC sont fréquentes en Afrique et demeurent un problème de santé publique malgré les progrès réalisés en vue de leur prévention (OMS, 2009 ; Barro et al., 2002). Ainsi, plusieurs auteurs ont estimé que, sur le milliard et demi d'épisodes diarrhéiques aigus annuels survenus dans la population des enfants de moins de cinq ans, $70 \%$ sont dus à des aliments contaminés (OMS, 2009 ; Batz, 2005 ; Berche, 2001). En outre, les denrées alimentaires dangereuses entraînent 1,2 millions de décès par an chez les personnes âgées de plus de cinq ans en Asie du Sud et en Afrique, et trois fois plus chez les adultes selon le rapport de l'OMS (2009).

La manipulation des denrées alimentaires par des personnes porteuses de microorganismes pathogènes, et l'utilisation inadéquate des antibiotiques aussi bien en médecine humaine que vétérinaire favorisent l'émergence des BMR qui peuvent se propager des animaux vers les humains par le biais des aliments, surtout au cours de la restauration collective avec, à la clé, des conséquences sanitaires et environnementales majeures immédiates et/ou tardives (Masterton, 2008; Barza. et Potentia, 2002 ; Swart 2002).

Au Bénin, les TIAC ne sont pas rares, d'après Barro et al. (2002) et Chauliac et al. (1998). Selon ces auteurs, leur fréquence est largement sous-évaluée par les autorités sanitaires. Leurs origines sont rarement élucidées du fait de la faiblesse des moyens de diagnostic, notamment les moyens microbiologiques (Fayomi et al., 1992).

Cette étude a pour objectif d'analyser la qualité bactériologique de trois aliments fréquemment vendus dans les cantines de l'Université d'Abomey-Calavi (UAC), et de déterminer la résistance des souches microbiennes isolées aux antibiotiques couramment prescrits en clinique au Bénin.

\section{MATERIEL ET METHODES \\ Nature et période d'étude}

Nous avons réalisé une étude prospective sur six mois; de la période allant du 09 septembre 2006 au 15 avril 2007 avec une interruption d'un mois (du 15 décembre au 14 janvier 2007) sur le campus de l'UAC, la plus grande université du Bénin dont la population estudiantine est estimée par le Service de la Scolarité du Rectorat à environ 45000 au moment de la réalisation de notre étude.

\section{Collecte des prélèvements alimentaires}

Deux comptoirs différents de «restauration rapide» très fréquentés ont été choisis et trois types d'aliments ont été ciblés. Un échantillonnage représentatif à la fois qualitatif et quantitatif des différents aliments sélectionnés a permis de collecter 390 échantillons d'aliments: Cent (100) morceaux de sandwich (tomates fraiches, mayonnaise, viandes hachées de bœuf, oignons émincés dans une tranche de pain de $100 \mathrm{~g}$ ); puis 120 portions du premier plat qui est composé de pâte de maïs accompagné de poisson d'eau douce en sauce tomate additionné de feuilles de crincrin (Corchorus olitoris); enfin 120 portions du deuxième plat ou monyô composé de pâte de maïs fermenté accompagné de poisson ou de viande de mouton assaisonné de tomate- monyô-, additionné de piment vert moulu et d'oignon émincé. Les boissons qui accompagnent le plus souvent ces aliments ont été également testées. Il s'agit de 50 prélèvements (25 de citronnade sucrée et glacée et 25 d'eau de robinet). Les aliments achetés sont transférés dans des conditions d'asepsie rigoureuse dans des sachets stériles 
et transportés dans des emballages calorifugés. L'ensemencement est réalisé dans l'intervalle d'une heure. Les prélèvements sont gardés au congélateur à $-20{ }^{\circ} \mathrm{C}$.

\section{Analyse bactériologique des prélèvements}

Les différents prélèvements d'aliments et de boissons collectés ont été analysés pour leur qualité bactériologique par dénombrement total direct des bactéries à 35 ${ }^{\circ} \mathrm{C}$, méthode précédemment décrite par le Centre d'Expertise du Québec (2005). Nous avons également joué sur la température pour différencier les bactéries thermo tolérants des coliformes fécaux selon Leclerk (1990). De manière spécifique, deux bactéries entéro pathogènes: E. coli et Salmonella ainsi que deux bactéries résistantes Staphylococcus aureus (S. aureus) résistante à la méticicilline (SARM) et les Entérocoques résistantes à la vancomycine $(E R V)$ ont été recherchées. Dix (10) g de chaque prélèvement sont pesés, et finement découpés puis mélangés. Un (1) g de ce mélange est ajouté respectivement à $9 \mathrm{~mL}$ d'eau peptonée stérile, et à du bouillon Rapapport. Les suspensions d'aliments obtenues sont homogénéisées à l'aide d'un appareil ultra- turax (IKA Labortechnik). Puis, $50 \mu 1$ de suspensions de chaque échantillon sont ensemencés sur les milieux suivants: Rapid' Ecoli pour la recherche de E coli, Slanetz pour la recherche des Streptocoques et le Baird Parker pour la recherche de Staphylocoque. A partir du bouillon Rapapport-Vassiliadis, la gélose Hektoen a été ensemencée pour la recherche des souches de Salmonella. Les boîtes ensemencées sont déposées à l'étuve à la température de $37{ }^{\circ} \mathrm{C}$ pendant 48 heures suivies d'une numération des bactéries. L'identification a été faite par les méthodes biochimiques classiques. Nous avons utilisé les normes béninoises et celles proposées par Jayarao, rapportées par Houser et al. (2008) pour évaluer la qualité bactériologique des aliments.

\section{Recherche des souches de $\boldsymbol{E}$. coli 0157}

L'isolement des colonies a été fait sur le milieu sorbitol MacConkey agar (oxoid CM
813) après pré enrichissement et séparation immunomagnétique. Les colonies sorbitol négatives ont été isolées et soumises aux tests d'identification par la galerie API 20 EC (API system réf 2045) et par agglutination avec un antisérum (DR0120M, Oxoid) permettant l'identification présomptive des souches de $E$. coli $\mathrm{O} 157$ suivant le protocole recommandé par Boer et al. (2001) et Boyce et al. (1995).

\section{Sensibilité des souches isolées aux antibiotiques \\ Un antibiogramme a été réalisé pour} chaque souche isolée par la méthode de diffusion à partir de disques chargés sur gélose de Mueller Hinton (Diagnostics Pasteur, Marnes la Coquette, France), selon les recommandations du NCCLS (2002). Les antibiotiques testés pour les entérobactéries sont: amoxicilline, amoxicilline-clavulanate $(20 / 10 \mu \mathrm{g}$,$) , céfoxitine (30 \mu \mathrm{g})$, céfotaxime (30 $\mu \mathrm{g})$, ceftazidime $(30 \mu \mathrm{g})$, ceftriaxone $(30 \mu \mathrm{g})$ aztréonam $(30 \mu \mathrm{g})$, ciprofloxacine $(5 \mu \mathrm{g})$, triméthoprime- sulfaméthoxazole $(1,25 / 23,75$ $\mu \mathrm{g})$. La souche d'E coli ATCC 25922 a servi de souche témoin pour le contrôle de qualité.

Pour les souches de $S$. aureus et d'entérocoques, les antibiotiques testés sont les suivants : penicilline $\mathrm{G}(6 \mu \mathrm{g})$, oxacilline $(5 \mu \mathrm{g})$, vancomycine $(30 \mu \mathrm{g})$, rifampicine (30 $\mu \mathrm{g})$, chloramphénicol (30 $\mu \mathrm{g})$, gentamicine $(15 \mu \mathrm{g})$, ciprofloxacine $(5 \mu \mathrm{g})$, erythromycine $(15 \mu \mathrm{g})$ et tétracycline $(10 \mu \mathrm{g})$.

\section{Détection de la bêtalactamase à spectre élargi parmi les souches de $E$. coli}

Toutes les souches ont été testées pour la production de la BLSE en utilisant en parallèle le test à la nitrocéfine et la méthode du double halo précédemment décrite par Jarlier et al. (1988), puis Thomson et al. (1992).

\section{Recherche de la production des entérotoxines par les souches de $S$. aureus \\ Les entérotoxines staphylococciques dans les aliments ont été détectées par dosage immunoenzymatique au moyen de la trousse}


TECKRA (International Bioproducts, Inc) comme décrit par Park et al. (1996).

\section{Méthode de comparaison statistique}

Les données ont été encodées et analysées avec le logiciel SPSS version 10.1. Les différentes proportions ont été comparées par les tests de $\chi 2$ de Pearson ou de Fischer au seuil de signification de 0,05 .

\section{RESULTATS}

\section{Caractéristiques bactériologiques des} aliments analysés

En six (6) mois, 390 prélèvements d'aliments ont été collectés. Il s'agit de 340 prélèvements de mets et 50 prélèvements de boissons. En effet $74 \%$ sont contaminés par des bactéries indicatrices d'altération générale (Tableau 1). Les fréquences de contamination de $38 \%$ par E. coli et de $18 \%$ par les entérocoques attestent d'une contamination d'origine fécale (Tableau 2). Nous avons observé également la présence de bactéries pathogènes: S. aureus (27\%) et Salmonella enterica enterica $(0,5 \%)$. A cela, nous pouvons ajouter cinquante (50) souches de $S$. sciuri isolées majoritairement dans le monyô et l'eau de boisson dont la fréquence ne figure pas parmi les résultats du Tableau 2.

\section{Prévalence de $\boldsymbol{E}$. coli 0157 dans les aliments} Nous avons identifié 21 souches de $E$. coli 0157 sur 147 E. coli (21/147) soit $14 \%$, réparties respectivement $8 \%(8 / 100)$ dans le sandwich et $11 \%(13 / 120)$ dans le monyô.

\section{Production des entérotoxines staphylococciques}

Les entérotoxines staphylococciques sont produites par 55 souches de $S$. aureus sur les 106 isolées soit $52 \%$.

\section{Sensibilités aux antibiotiques des souches isolées}

Sur 325 bactéries testées, 116 (soit 36\%) se sont révélées résistantes à plus de 5 antibiotiques et constituent les BMR réparties pour $44 \%$ de ECBLSE, $32 \%$ de SARM et $24 \%$ d'ERV. La résistance au triméthoprimesulfaméthoxazole et à la tétracycline, dépasse 50\%; la Figure 1 et le Tableau 3 illustrent les différents profils de résistance obtenus par grande famille d'antibiotiques.

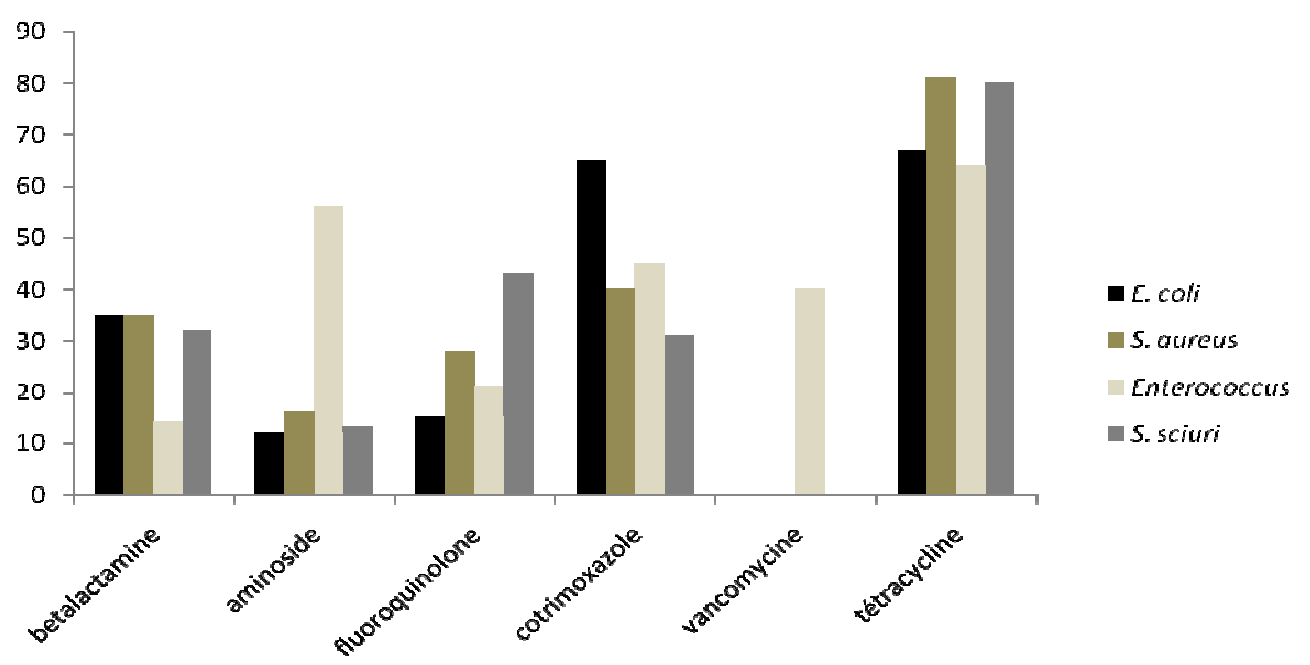

Figure 1: Profil de résistance des bactéries aux grandes familles d'antibiotiques. 
Tableau 1: Qualité bactériologique des mets et boissons analysés par dénombrement total.

\begin{tabular}{|c|c|c|c|c|}
\hline $\begin{array}{l}\text { Echantillons } \\
\text { D'aliments }\end{array}$ & $\begin{array}{l}\text { Numération } \\
\text { UFC/mL }\end{array}$ & $\begin{array}{l}\text { Coliformes } \\
\text { Fécaux }\end{array}$ & $\begin{array}{l}\text { Coliformes } \\
\text { Thermotolérants }\end{array}$ & $\begin{array}{c}\text { Valeur de P, } \\
\text { Test de Fisher }\end{array}$ \\
\hline \multirow{3}{*}{$\begin{array}{c}\text { Sandwich } \\
100\end{array}$} & Moyenne (SD) & $179 \pm 111$ & $53 \pm 12$ & \multirow{3}{*}{$\mathrm{P}<0,001$} \\
\hline & minima & 0 & 0 & \\
\hline & Maximum & 1080 & 105 & \\
\hline \multirow{3}{*}{$\begin{array}{c}\text { Pâte + sauce tomate } \\
n=120\end{array}$} & Moyenne & $206 \pm 99$ & $72 \pm 21$ & \multirow{3}{*}{$\mathrm{P}<0,0001$} \\
\hline & minima & 0 & 0 & \\
\hline & Maximum & 695 & 120 & \\
\hline \multirow{3}{*}{$\begin{array}{c}\text { Akassa+ } \\
\text { Monyô } n=120\end{array}$} & Moyenne & $426 \pm 253$ & $94 \pm 31$ & \multirow{3}{*}{$\mathrm{P}<0,0001$} \\
\hline & minima & 0 & 0 & \\
\hline & Maximum & 2000 & 170 & \\
\hline \multirow{3}{*}{$\begin{array}{c}\text { Boisson } \\
n=50\end{array}$} & Moyenne & $332 \pm 174$ & $101 \pm 45$ & \multirow{3}{*}{$\mathrm{P}<0,0001$} \\
\hline & minima & 0 & 0 & \\
\hline & Maximum & 4590 & 230 & \\
\hline \multirow[t]{3}{*}{ Total } & Moyenne & $635 \pm 123$ & $121 \pm 23$ & \multirow{3}{*}{$\mathrm{P}<0,0001$} \\
\hline & minima & 0 & 0 & \\
\hline & Maximum & 12000 & 6800 & \\
\hline
\end{tabular}

UFC: unité formant colonie

Tableau 2: Qualité bactériologique et fréquence de contamination des aliments par des bactéries pathogènes.

\begin{tabular}{|c|c|c|c|c|c|c|c|c|c|}
\hline \multirow[t]{2}{*}{ Echantillons } & \multicolumn{2}{|c|}{ S aureus } & \multicolumn{2}{|c|}{ Entérocoque } & \multicolumn{2}{|c|}{ E coli } & \multicolumn{2}{|c|}{ Salmonella } & \multirow{2}{*}{$\begin{array}{c}\text { Aliments } \\
\text { sains, } \\
\text { Nombre et \% }\end{array}$} \\
\hline & $>0$ & 0 & $>0$ & 0 & $>0$ & 0 & $>0$ & 0 & \\
\hline Sandwich 100 & 43 & 57 & 20 & 80 & 24 & 76 & 0 & 100 & $45 \%$ \\
\hline $\begin{array}{l}\text { Pâte + sauce } \\
\text { tomate } 120\end{array}$ & 27 & 93 & 15 & 105 & 31 & 89 & 0 & 120 & $40(33 \%)$ \\
\hline $\begin{array}{l}\text { Akassa+Monyo } \\
120\end{array}$ & 23 & 97 & 25 & 95 & 71 & 49 & 0 & 120 & $7(6 \%)$ \\
\hline Boisson 50 & 13 & 37 & 10 & 40 & 21 & 29 & 2 & 48 & $8(16 \%)$ \\
\hline Total 390 & 106 & 284 & 70 & 320 & 147 & 253 & 2 & 388 & $\begin{array}{c}100 / 390 \\
(26 \%)\end{array}$ \\
\hline
\end{tabular}

Aucun de ces microorganismes ne doit exister dans les aliments à consommer. Le monyô akassa est le met le plus contaminé 
Tableau 3: Proportion de souches résistantes aux antibiotiques par espèce bactérienne isolée dans les aliments analysés.

\begin{tabular}{lcccccc}
\hline $\begin{array}{l}\text { Nature des } \\
\text { aliments }\end{array}$ & $\begin{array}{c}\text { Nombre } \\
\text { de } \text { E. coli }\end{array}$ & $\begin{array}{c}\text { \% ECBLSE } \\
\text { dans E. coli }\end{array}$ & $\begin{array}{c}\text { Nombre de } \\
\text { S. aureus }\end{array}$ & $\begin{array}{c}\text { \% SARM/ } \\
\text { S. aureus }\end{array}$ & $\begin{array}{c}\text { Nombre de } \\
\text { Enterococcus }\end{array}$ & \% ERV \\
\hline Sandwich, n=100 & 24 & $14(58 \%)$ & 43 & $11(26 \%)$ & 20 & $12(60 \%)$ \\
$\begin{array}{l}\text { Pâte + sauce } \\
\text { tomate, } \mathrm{n}=120\end{array}$ & 31 & $4(13 \%)$ & 27 & $9(33 \%)$ & 15 & $2(13 \%)$ \\
$\begin{array}{l}\text { Akassa+ } \\
\text { Monyô, n=120 }\end{array}$ & 71 & $30(21 \%)$ & 23 & $13(57 \%)$ & 25 & $10(20 \%)$ \\
$\begin{array}{l}\text { Boisson, n=50 } \\
\text { Total }\end{array}$ & 21 & $3(10 \%)$ & 13 & $6(46 \%)$ & 10 & $4(40 \%)$ \\
\hline
\end{tabular}

\section{DISCUSSION}

L'objectif de notre étude est d'analyser la qualité microbiologique des aliments vendus en restauration rapide sur le plus grand campus universitaire du Bénin, et de déterminer parmi les souches microbiennes isolées celles qui sont multi-résistantes aux antibiotiques. Les résultats obtenus au cours de notre étude révèlent une fréquence relativement élevée de $74 \%$ d'aliments contaminés par une ou plusieurs bactéries en comparaison avec les différentes études réalisées dans les pays industrialisés, où des fréquences moyennes variant de 5 à $25 \%$ ont été rapportées par plusieurs auteurs (OMS, 2005 ; Berche, 2001; Mead et al., 1999; Todd, 1996). Nos résultats sont semblables aux proportions trouvées dans les pays africains à taux élevés d'aliments insalubres, comme celui obtenu par Drabo et al. (2008) ainsi que Barro et al. (2002). La fréquence de contamination de $69 \%$ trouvée dans le lait cru par Houser et al. (2008) en Pennsylvanie et celle de $83 \%$ des crèmes et glaces rapportée par Kruy et al. (2001) à Phnom Penh semblent révéler les mêmes réalités. La plupart des bactéries isolées ont conservé leur vitalité et leur pouvoir pathogène et se retrouvent en nombre suffisamment important dans les mets analysés pour provoquer des maladies (voir les données du Tableau 1).

Les coliformes fécaux et les entérocoques sont très présents dans les aliments par rapport aux coliformes thermotolérants avec une différence statistiquement significative $(\mathrm{p}<0,001)$. E. coli est la bactérie la plus isolée avec $14 \%$ de $E$. coli $\mathrm{O} 157$ qui non seulement est capable de croître à $4{ }^{\circ} \mathrm{C}$, mais est acido-tolérante et résistante à la température de $60{ }^{\circ} \mathrm{C}$. Plusieurs épidémies directement causées par ce sérotype ont été signalées dont $52 \%$ sont attribuées aux aliments mal cuits selon Elder et al. (2000) ainsi que Bauda et Monfort (2004). Le monyô est l'aliment le plus contaminé (94\%) par les bactéries dans notre étude, puis la pâte +sauce $(67 \%)$ suivi du sandwich (55\%). Ces résultats ne sont pas étonnants à cause de la composition et du mode de préparation de ces aliments où la température de cuisson est à peine supérieure à $60^{\circ} \mathrm{C}$.

S. aureus est la deuxième bactérie isolée parmi lesquelles $52 \%$ sont sécrétrices d'au moins une des sept entérotoxines staphylococciques. Ces mets contaminés sont susceptibles de provoquer des TIAC à staphylocoques chez les consommateurs, une maladie courte mais éprouvante et spectaculaire, pouvant prendre une allure 
dramatique lorsqu'elle atteint une collectivité comme la communauté estudiantine. La présence de $S$. scuiri, un staphylocoque d'origine animale, dans notre enquête, est un indice supplémentaire qui plaide pour la contamination de mets que nous avons analysés; car la transmission de ces microorganismes est très souvent manuportée à partir de réservoirs essentiellement humains. Nous avons observé également des souches de Salmonelle, bactéries pathogènes à l'origine des intoxications alimentaires comme c'est aussi le cas de $S$. aureus. Ces contaminations proviendraient probablement de mauvaises pratiques de préparation des denrées alimentaires ou de souillures d'origine fécale lors de manipulations par des mains sales. Il a été précédemment rapporté par Chambers (2002) que la qualité bactériologique du lait pouvait directement être liée aux conditions d'hygiène mises en jeu au cours du traitement. Nous pensons que ces mêmes observations peuvent s'appliquer aux aliments que nous avons analysés et peuvent se résumer en un manque d'hygiène, de protection et de conservation de ces aliments.

Dans notre étude, $36 \%$ des bactéries isolées développent une résistance à plus de cinq antibiotiques et constituent des BMR comme l'illustre la Figure 1, ce que corrobore l'étude de Megan et al. (2008). Les antibiotiques les plus touchés sont la vancomycine, la tétracycline, l'ampiciline, le triméthoprime-sulfaméthoxazole, les fluoroquinolones qui sont couramment prescrits en médecine humaine au Bénin. Les BMR sont souvent isolées en milieu hospitalier avec des fréquences de 12 à 28,7\% rapportées par Ahoyo et al. (2007) et sont responsables des infections nosocomiales sévères; mais aujourd'hui, nous assistons à leur prolifération dans la communauté, surtout dans les denrées alimentaires avec des fréquences de $58 \%$ et de $88 \%$ au sein des bactéries indicatrices d'une contamination fécale; respectivement trouvées par Persoons et al. (2010) en Belgique, puis Megan et al. (2008) avec une possible transmission des souches d'origine animale vers les humains à travers l'alimentation. Nos résultats sont du même ordre de grandeur que les données hospitalières et sont inquiétantes car elles posent un réel problème de santé du monde estudiantin et de toute la communauté universitaire.

L'émergence des BMR serait le résultat de l'utilisation massive des antibiotiques en médecine vétérinaire, comme facteurs de croissance des animaux, mais également dans diverses activités agricoles des pays industrialisés selon Masterton (2008) et Aarestrup (2005). Aussi, Wauters (1992) a-t-il évoqué l'usage abondant des céphalosporines et de la vancomycine en médécine humaine pour expliquer la résistance des entérocoques à ces antibiotiques. Tous ces facteurs ont contribué à une augmentation alarmante de la résistance des microorganismes aux antimicrobiens. Dans la plupart des pays africains, la sécurité alimentaire n'est pas garantie, de sorte qu'au moins $60 \%$ des denrées alimentaires sont importées pour compléter la production locale selon Dorandeu (2008). La plupart de ces pays n'ont pas de dispositif efficace de contrôle, d'où un risque accru de contamination à grande échelle. L'ensemble de ces facteurs explique la détérioration de la qualité des aliments analysés selon Barbano et al. (2006). Par ailleurs, nous avons constaté au cours de la collecte des échantillons que les mets étaient préparés tôt le matin et conservés à la température ambiante tropicale jusqu'à épuisement du stock, parfois tard le soir. Ce sont autant de conditions propices à la prolifération des microorganismes dans les aliments. Selon l'opinion des vendeuses des mets incriminés, ce manque d'hygiène constaté dans la manipulation des aliments a son origine dans le mauvais état des installations d'assainissement et des équipements de ces cantines plutôt que dans une quelconque carence éducative

La pratique correcte du lavage des mains au savon dans les cantines reste faible malgré les différentes campagnes de sensibilisation et pourrait justifier en partie les résultats obtenus. 
L'implication des vendeuses dans la contamination des aliments a été suspectée dans $32 \%$ des cas, mais n'a été confirmé que dans 4 cas déterminés par typage selon la technique de l'électrophorèse en champ pulsé et fera l'objet d'une publication prochaine. L'analyse bactériologique périodique des prélèvements (selles, mains et narines) provenant des personnes impliquées dans la préparation et la distribution des aliments, de même que des échantillons de ces aliments pourrait faciliter la démonstration de l'implication de ces vendeuses. La connaissance de ces risques alimentaires impose de renforcer l'information sur le respect strict des conditions d'hygiène tout au long de la chaîne alimentaire depuis la préparation des aliments jusqu'à leur mise à disposition du consommateur.

\section{Limite de l'étude}

Le recueil des données de l'enquête ne permet pas d'appréhender le mode de contamination des aliments, ni d'incriminer l'un de ses constituants comme responsable de la contamination initiale. S'agit-il de l'eau utilisée, des matériels de préparation ou des manipulateurs? Il serait intéressant de pouvoir étudier plus méthodiquement les différentes phases de préparation pour déterminer les points critiques pouvant faire l'objet d'une surveillance particulière.

\section{Conclusion}

Cette étude nous a permis de montrer, outre la présence de E coli $\mathrm{O} 157$ dans certains aliments analysés, une fréquence élevée de BMR dans les aliments vendus dans les comptoirs de restauration rapide à l'UAC. L'association de plusieurs facteurs favorisants rend inévitable cette prolifération de microorganismes. La faible intervention $\mathrm{du}$ laboratoire de bactériologie dans le contrôle de qualité des denrées alimentaires, le bas niveau du respect de l'hygiène expliquent les résultats obtenus dans cette étude. La technique de cuisson des aliments dans notre contexte élimine souvent la majorité des microorganismes présents dans les denrées de départ. Par contre, les manipulations de ces aliments et les conditions de leur conservation constituent des sources de contamination. Ces données nous incitent à encourager le respect des mesures d'hygiène mais aussi et surtout la promotion du lavage des mains au savon pour limiter la contamination des aliments en restauration collective.

L'utilisation rationnelle des antibiotiques constitue un point essentiel à promouvoir pour lutter contre l'émergence des micro-organismes résistants qui représentent un risque présent et futur non seulement pour la personne exposée à un traitement, mais pour la population en général.

\section{REMERCIEMENTS}

Nous remercions les vendeuses des cantines, et les étudiants qui ont accepté de participer à cette enquête. Dr BANKOLE Honoré et $\mathrm{Mr}$ LEGONOU Magloire sont remerciés pour leur contribution dans la réalisation pratique $\mathrm{du}$ travail et les discussions utiles.

\section{REFERENCES}

Aarestrup FM. 2005. Veterinary drug usage and antimicrobial resistance in bacteria of animal origin. Basic Clin Pharmacol Toxicol., 96: 271-281.

Ahoyo AT, Baba-Moussa L, Anago AE, Avogbe P, Missihoun TD, Loko F, Prévost G, Sanni A, Dramane K. 2007. Incidence d'infections liées à Escherichia coli producteur de bêta lactamase à spectre élargi au Centre Hospitalier Départemental du Zou et Collines au Bénin. Médecine et Maladies Infectieuses, 37: 746-752.

Barbano DM, Ma Y, Santos MV. 2006. Influence of raw milk quality on fluid milk shelf life. J. Dairy Sci,, 89(E): E15E19.

Barro N, Ouattara Ca, Nikiema Pa, Ouattara AS, Traore AS. 2002. Microbial quality assessment of some street food widely consumed in Ouagadougou, Burkina Faso. Santé, 12: 369-374. 
Barza M, Potentia. 2002. Mechanisms of increased disease in humans from antimicrobial resistance in foods. Clinical Infections Disease, 34(3): 123-125.

Batz MB, Doyle MP, Morris GJr, Painter J, Singh R. 2005. Attributing illness to food. Emerg Infect Dis., 11: 993-999.

Bauda P, Monfort P. 2004. Agents pathogènes et modifications des environnements: quels risques actuels et futurs? Environnement, Risques et Santé, 3(3) : 165-72.

Berche P. 2001. L'émergence de nouveaux risques infectieux d'origine alimentaire. Annales de Biologie Clinique, 59(5): 585591.

Boere DE, Heuvelink AE. 2001. Evaluation of methods for the detection and isolation of Escherichia coli $\mathrm{O} 157$ from foods and faeces. Proceedings of European Union Concerted Action CT98-3935. Verocytotoxigenic E. coli in Europe. 1. Methods for Verocytotoxigenic E. coli, Duffy G, Garvey P, Coia J, Wasteson Y, McDowell DA (eds). Int. J. Food Microbiol., 66: 25-35.

Boyce TG, Pemberton AG, Wells JG, Griffin PM. 1995. Screening for Escherichia coli O157:H7- a Nationwide Survey of Clinical Laboratories. J. Clin. Microbiol., 33: $3275-3277$.

Centre D'expertise en Analyse Environnementale du Québec. 2005. Recherche et dénombrement des bactéries hétérotrophes aérobies et anaérobies facultatives : méthode par incorporation à la gélose. MA. 700 - BHA35 1.0, Rév. 2, Ministère du Développement durable, de l'Environnement et des Parcs du Québec, $15 \mathrm{p}$.

Chambers JV. 2002. The microbiology of raw milk. In Dairy Microbiology Handbook, (3rd edn), Robinson RK (ed). John Wiley \& Sons, Inc: New York; 39-90.

Chapman B, Eversley T, Fillion K, MacLaurin T, Powell D. 2010. Assessment of Food Safety Practices of Food Service Food Handlers: Testing a Communication
Intervention. Journal of Food Protection, 73(6): 1101-1107.

Chauliac M, Bricas N, Ategbo E, Amoussa W, Zohoun I. 1998. Food habits outside the home by school children in Cotonou (Benin). Santé, 8:101-108.

Dorandeu F. 2008. Risques toxiques dans les pays à infrastructures déficitaires. Médecines Tropicales, 68(4): 375-384.

Drabo KM, Pare Toe, Savadogo LGB, Tarnagda Z, Zeba AN, Zongo I, Rouamba J, Toe A, Ouédraogo D, Ouédraogo JB. 2009. Caractéristiques de l'alimentation de rue dans la ville de Bobo-Dioulasso, Burkina Faso. Bull Soc Pathol Exot, 102(1): 36-40.

Elder RO, James EK, Gregory RS, GallagherBarkocy GA, Koohmaraie M, Laegreid WW. 2000. Correlation of enterohemorrhagic E. coli O157 prevalence in feces, hides, and carcasses of beef cattle during processing. Proc. Natl. Acad. Sci., 97: 2999-3003.

Fayomi B, Josse R, Adaye A, Laloe V, Djogbe H, Zohoun R. 1992. Toxiinfection alimentaire en milieu rural béninois. Médecine d'Afrique Noire, 39(5): 364-366.

Houser B, Donaldson S, Kehoe Heinrichs, Jayarao BM. 2008. A Survey of Bacteriological Quality and the Occurrence of Salmonella in Raw Bovine Colostrum. Foodborne Pathogens and Disease, 5(6): 853-858.

Jarlier V, Nicolas M, Fournier G, Philippon A. 1988. Extended broadspectrum $\beta$ lactamases conferring transferable resistance to newer $\beta$-lactam agents in Enterobacteriaceae: hospital prevalence and susceptibility patterns. Rev. Infect. Dis. 10: 867-878.

Kruy SL, Soares JL, Ping SF, Flye S-M. 2001. Qualité microbiologique de l'aliment "glace, crème glacée, sorbet" vendu dans les rues de la ville de Phnom Penh; avril 1996 - avril 1997. Bull Soc Pathol Exot., 94(5): 411-414.

Leclerk H 1990 : indicateurs bactériens et contrôle de qualité des eaux minérales 
naturelles. Revista Italiana d'igiene, 5(6): 363-365.

Masterton R. 2008. The importance and future of antimicrobial surveillance studies. Clin. Infect. Dis., 47(1): 21-31.

Mead PS, Slutsker L, Dietz V, McCaig LF, Bresee JS, Shapiro C, Griffin PM, Tauxe RV. 1999. Food-related illness and death in the United States. Emerg. Infect. Dis., 5: 607-625.

Megan EJ, Trent Fox J, Reinstein SL, Nagaraja TG. 2008. Antimicrobial susceptibility of foodborne pathogens in organic or natural production systems: An overview. Foodborne Pathogens and Disease, 5(6): 721-730.

National Committee for Clinical Laboratory Standards. 2002. Performance standards for antimicrobial susceptibility testing, 12th informational supplement. Wayne, Pa: NCCLS document M100-S12.

OMS (Organisation Mondiale de la Santé). 2005. Premier colloque régional sur la sécurité alimentaire en Afrique rapport OMS (12 octobre 2005).

OMS (Organisation Mondiale de la Santé). 2009. Sécurité sanitaire des aliments; Bulletin publié par le Département Sécurité sanitaire des aliments et zoonoses FOS No 35 - 12 novembre 2009.

Park CE, Warbuton DW, Laffey PJ. 1996. A collaborative study on the detection of staphylococcal enterotoxin in foods with an enzyme immunoassay kit (TECRA). $J$. Food Prot., 59: 390-397.

Persoons D, Dewulf J, Smet A, Herman L, Heyndrickx M, Martel A, Catry B, Butaye P, Haesebrouck F. 2010. Prevalence and persistence of antimicrobial resistance in broiler indicator bacteria. Microbial Drug Resistance, 16(1): 67-74.

Sharma S, Sachdeva P, Virdi JS. 2003. Emerging waterborne pathogens. Appl Microbiol Biotechnol, 61: 424-428.

Swart MN. 2002. Human diseases caused foodborne pathogens of animal origin. Clinical Infections Disease, 34(3): 111122.

Thomson KS, Sanders CC. 1992. Detection of extended-spectrum $\quad \beta$-lactamase in members of the family Enterobacteriaceae: comparison of the double-disk and three-dimensional tests. Antimicrobial Agents Chemother, 36: 1877-1882.

Todd ECD. 1996. Intoxications alimentaires et maladies d'origine hydrique au Canada: sommaires annuels: 1988 à 1989. Ottawa (Ont.): Direction générale de la protection de la santé, (Ministère des Approvisionnements et Services, ISBN 0921317-58-1).

Wauters G. 1993. les entérocoques dans hygiène hospitalière. Bulletin d'Information, XV(1): 1- 4. 